Improved accuracy in the determination of flexural rigidity of textile fabrics by the Peirce cantilever test (ASTM D1388)

Nicolas Lammens, Mathias Kersemans, Geert Luyckx, Wim Van Paepegem and Joris Degrieck Textile Research Journal published online 13 February 2014

DOI: $10.1177 / 0040517514523182$

The online version of this article can be found at:

http://trj.sagepub.com/content/early/2014/02/12/0040517514523182

Published by:

(9)SAGE

http://www.sagepublications.com

Additional services and information for Textile Research Journal can be found at:

Email Alerts: http://trj.sagepub.com/cgi/alerts

Subscriptions: http://trj.sagepub.com/subscriptions

Reprints: http://www.sagepub.com/journalsReprints.nav

Permissions: http://www.sagepub.com/journalsPermissions.nav

>> OnlineFirst Version of Record - Feb 13, 2014

What is This? 


\title{
Improved accuracy in the determination of flexural rigidity of textile fabrics by the Peirce cantilever test (ASTM DI388)
}

Textile Research Journal $0(00) \mathrm{I}-8$

(C) The Author(s) 2014

Reprints and permissions: sagepub.co.uk/journalsPermissions.nav DOI: 10.1 | 77/00405|75|4523|82 trj.sagepub.com SAGE

\author{
Nicolas Lammens, Mathias Kersemans, Geert Luyckx, \\ Wim Van Paepegem and Joris Degrieck
}

\begin{abstract}
Within the field of composite manufacturing simulations, it is well known that the bending behavior of fabrics and prepregs has a significant influence on the drapeability and final geometry of a composite part. Due to sliding between reinforcements within a fabric, the bending properties cannot be determined from in-plane properties and a separate test is required. The Peirce cantilever test represents a popular way of determining the flexural rigidity for these materials, and is the preferred method in the ASTM DI388 standard. This work illustrates the severe inaccuracies (up to $72 \%$ error) in the current ASTM DI388 standard as well as the original formulation by Peirce, caused by ignoring higher-order effects. A modified approach accounting for higher-order effects and yielding significantly improved accuracy is presented. The method is validated using finite element simulations and experimental testing. Since no independent tests other than the ASTM DI 388 standard are available to determine the bending stiffness of fabric materials, experimental validation is performed on an isotropic, homogeneous Upilex-50S foil for which the flexural rigidity and tensile stiffness are related. The flexural rigidity and elastic modulus are determined through both the cantilever test (ASTM DI388) and tensile testing. The results show that the proposed method measures an elastic modulus close to that determined through tensile testing (within 1\%), while both the Peirce formulation (+18\%) and ASTM standard (+72\%) over-estimate the elastic modulus. The proposed methodology allows for a more accurate determination of flexural rigidity, and enables the more accurate simulation of composite forming processes.
\end{abstract}

\section{Keywords}

testing, fabrication, properties, materials, textile care/wrinkling, management of systems, product and systems engineering

The increasing popularity of composite materials has led to the development of several simulation tools predicting the final shape and strength of a composite production process. ${ }^{1-5}$ When fabric reinforcements are used, an important part of this simulation tool is drape prediction. Fish-net algorithms have been developed, using the assumption of inextensibility along the reinforcing directions, and pivot-points where warp and weft cross. $^{6-9}$ These algorithms are capable of predicting basic drape behavior, without requiring any material parameters or accounting for tool-fabric interactions. As a consequence, however, these algorithms predict the same shape, regardless of the reinforcing material and constraints, limiting their usability and accuracy. ${ }^{4}$ A different approach is through the use of finite element analysis taking into account all important material parameters that dictate the forming capability. ${ }^{10-16}$ It is generally agreed that the parameters (i) tensile stiffness, (ii) shear stiffness (and its locking angle), (iii) tool-ply and inter-ply friction and (iv) bending stiffness are dominant factors in the

Department of Materials Science \& Engineering, Ghent University, Belgium

Corresponding author:

Nicolas Lammens, Department of Materials Science \& Engineering, Ghent University, Technologiepark-Zwijnaarde 903, 9052 Zwijnaarde, Belgium. Email: Nicolas.Lammens@ugent.be 
material model. ${ }^{1}$ In addition, it is known that the finite element simulation of fabric draping requires a specialized framework to follow the fiber directions. ${ }^{17}$ Several authors have presented different finite element implementations, incorporating a number of these parameters with good results. ${ }^{10-19}$

The comparison of finite elements with experimental results will, at some point, require the determination of the necessary material parameters. A couple of procedures for these tests were originally published by Peirce ${ }^{20}$ and later implemented as ASTM standards. $^{21}$ This work focuses on the determination of bending stiffness as described by Peirce and ASTM D1388-08. Hamila etal. ${ }^{13}$ have shown the influence of bending stiffness on predicted final geometry and wrinkling behavior in draping simulations. Based on finite element models, inaccuracies in both approaches are traced back, and a modified solution method is proposed. Both the ASTM and Peirce method are compared to the newly proposed method through experimental testing of samples of Upilex-50S foil. Although the ASTM standard is designed to be used with fabric materials, the fundamental equations on which the method relies were developed for any type of flexible material. ${ }^{22}$ In order to compare the absolute accuracy of the different methods, a reference value has to be determined through an independent test. Using fabric materials, the bending stiffness stated by the manufacturer would most likely be based on the ASTM standard discussed in this work. This would inevitably result in the incorrect conclusion that the ASTM standard achieves the most accurate results. As a consequence the tests have been conducted on an isotropic, homogeneous Upilex-50S material for which the bending stiffness can be related to the tensile modulus determined through tensile testing (ASTM D882). The choice for Upilex-50S was found to be sufficiently flexible to be tested in the cantilever test, while still having a sufficiently thick cross-section in order to be accurately tested in the tensile testing facilities available.

The experimental results illustrate the increased accuracy of the new method, yielding more accurate determination of the bending behavior, and as a consequence enabling a more accurate simulation of drape behavior in composite forming simulations.

\section{Peirce cantilever test}

The ASTM standard for determination of bending stiffness in fabric materials suggests the use of Peirce's cantilever test (Figure 1) as the preferred methodology to measure bending stiffness.

A specimen of the fabric is cut to the correct dimensions $(200 \mathrm{~mm} \times 25 \mathrm{~mm})$ and gradually slid over the

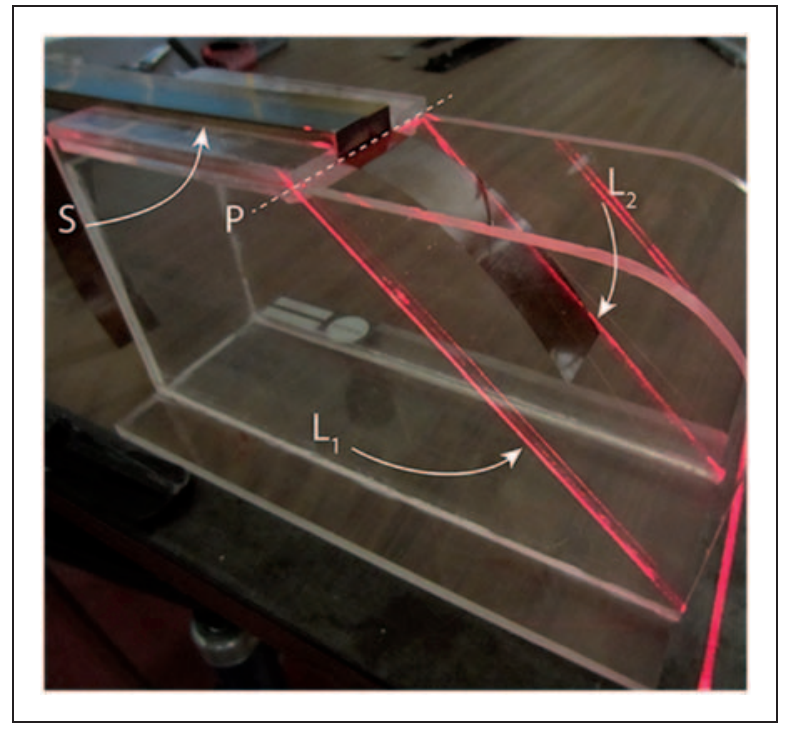

Figure I. Peirce cantilever device.

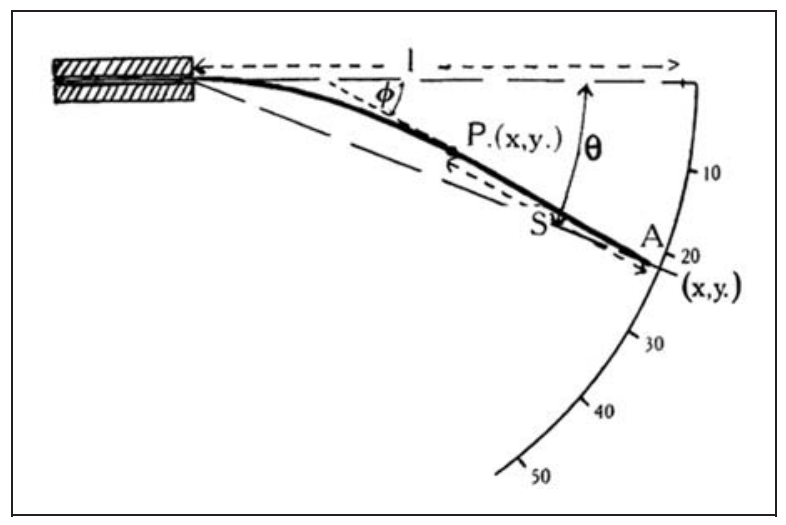

Figure 2. Schematic representation of Peirce cantilever test and relevant parameters. ${ }^{20}$

edge $(\mathrm{P})$ of the top-surface, until the leading edge of the fabric makes contact with the angled surface of the device $\left(\mathrm{L}_{1}, \mathrm{~L}_{2}\right)$. Using a graduated ruler $(\mathrm{S})$, the overhanging length $l$ of the specimen is measured (Figure 2). Within the ASTM standard, a fixed angle $\theta=41.5^{\circ}$ is used for the inclined surface. Some research has proposed to increase this angle to $\theta=43^{\circ}$, stating that this would increase the sensitivity of the method. ${ }^{23}$ The original formulas presented by Peirce can be modified to provide results for any inclination.

According to the original research paper by Peirce, the measured length $l(\mathrm{~mm})$ should then be multiplied with a factor $f_{1}(\theta)$ leading to the so-called 
bending length $c=l \cdot f_{1}(\theta)$. This scaling factor is determined as

$$
f_{1}(\theta)=\left(\frac{\cos \theta / 2}{8 \cdot \tan \theta}\right)^{1 / 3}
$$

The choice of $\theta=41.5^{\circ}$ within the ASTM standard is mainly related to practical aspects as for this angle $f_{1}\left(41.5^{\circ}\right) \approx 0.5$.

If $c$ has units of $\mathrm{mm}$ and the areal weight $\mathrm{w}$ is expressed in $\mathrm{g} / \mathrm{m}^{2}$, the flexural rigidity, in units of $\mathrm{N}$ $\mathrm{m}$ according to Peirce is found as

$$
G_{\text {Peirce }}=9.81 \cdot 10^{-12} \cdot w \cdot c^{3}
$$

The ASTM standard differs from the Peirce formulation in the addition of a (significant) scaling factor, for which no explanation is given. Using the same units for $c$ and $w$, the flexural rigidity according to ASTM is found as (units of $\mathrm{N} \mathrm{m}$ )

$$
G_{A S T M}=1.421 \cdot 10^{-11} \cdot w \cdot c^{3}
$$

Comparing Equations (2) and (3), it is easily found that $G_{A S T M}=1.45 \cdot G_{\text {Peirce }}$. As a result, applying both the ASTM and Peirce formulations, ASTM will consistently result in a bending stiffness, which is $45 \%$ higher than that according to Peirce.

The ASTM standard provides no details as to why this scale factor is introduced. In addition, according to this standard, $G$ is expressed in units of $\mu \mathrm{J} / \mathrm{m}$, equating to $\mu \mathrm{N}$ rather than the expected $(\mu) \mathrm{Nm}$. It should also be noted that this scale factor has only been introduced in the latest revisions of the standard (D1388- 08). The scale factor is not present in older versions of the standard.

\section{Finite element modeling of cantilever test}

Current computational power available in the average computer is more than sufficient to perform a finite element simulation of the cantilever bending experiment. A finite element model was created in ABAQUS in order to compare the finite element predictions to the analytical approaches proposed by Equations (2) and (3).

Since Equations (2) and (3) are essentially derived from beam theory, two models were built: one using beam elements, the other using S8R quadratic shell elements. Both models have an approximate element size of $0.25 \mathrm{~mm}$ (see Figure 3). The shell model has a fixed width of $20 \mathrm{~mm}$ in accordance with the ASTM standard procedure. The length of the part is swept between 25 and $200 \mathrm{~mm}$ and the resulting inclination

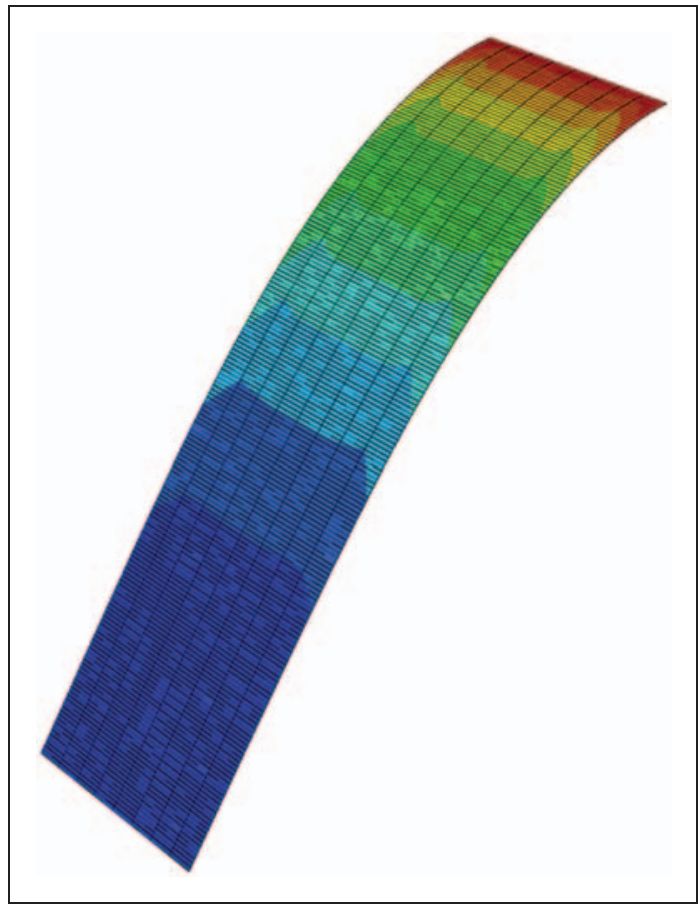

Figure 3. Finite element model of the Peirce cantilever test.

angle $\theta$ is calculated from the finite element results, giving the relation between $l$ and $\theta$.

The finite element analysis is performed on an isotropic Upilex-50S material. Upilex material is a polyimide foil material mainly used in high-temperature applications, such as aerospace. This material has a density of $\rho=1.47 \mathrm{~g} / \mathrm{cm}^{3}$, a theoretical thickness of $50 \mu \mathrm{m}$ and a tensile modulus of $E=8042 \mathrm{MPa}$ (interpolated between manufacturer-provided values for Upilex-25S and Upilex-75S, since no data is provided for Upilex-50S) and a Poisson's ratio of $v=0.25$. Using this data, we find an areal weight of $w=73.5 \mathrm{~g} / \mathrm{m}^{2}$, and assuming beam theory $G=\frac{E \cdot t^{3}}{12}=8.377 \cdot 10^{-5} \mathrm{Nm}$. Filling in these values in Equations (2) and (3), we find the relationship between $l$ and $\theta$ according to Peirce and ASTM, respectively.

Figure 4 shows the result of the finite element simulations, together with the ASTM and Peirce formulations.

These results show that at an angle $\theta=41.5^{\circ}$, the Peirce formulation is close to the finite element results assuming beam theory, while the ASTM standard predicts a significantly different length. The correspondence between Peirce and finite element beam theory becomes worse for larger angles. In addition, finite element predictions using beam and plate theory result in small differences, suggesting that plate effects might need to be taken into account. This is to be expected given the rather high width-to-length ratio 


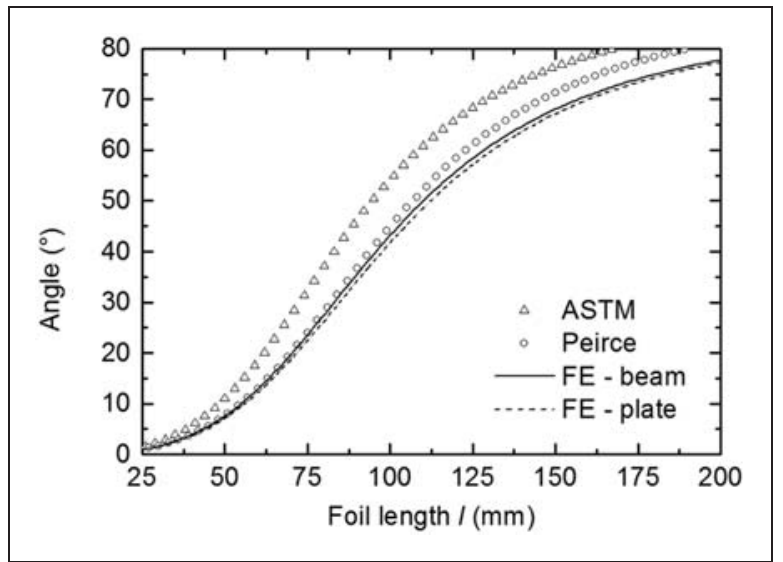

Figure 4. Bend angle versus overhanging length according to finite element simulations, Peirce and ASTM formulations for a Upilex-50S foil.

Table I. Overhanging length according to American Society for Testing and Materials (ASTM), Peirce and finite element (F.E.) for a Upilex-50S foil.

\begin{tabular}{lllll}
\hline & ASTM & Peirce & F.E. & \\
\cline { 3 - 4 } & & Beam theory & Plate theory \\
\hline $\mathrm{I}(\mathrm{mm})$ & 84.7 & 95.8 & 97.6 & 99.6 \\
\hline
\end{tabular}

of the samples as specified by the ASTM standard. Depending on the Poisson's ratio, beam assumptions might be invalid.

The exact overhanging lengths at $\theta=41.5^{\circ}$ are stated in Table 1. Even though Figure 4 suggests that finite element beam theory and Peirce closely match, Table 1 shows that a difference of $2 \mathrm{~mm}$ in overhanging length exists between both. An additional $2 \mathrm{~mm}$ difference exists between beam theory and plate theory for this specific material and geometry.

Note that the reverse process of starting from a known overhang length and angle and trying to determine the corresponding bending stiffness would require an iterative finite element process, which would hinder the practical applicability of using finite element techniques to determine the bending stiffness from a cantilever test.

\section{Revised Peirce equations}

\section{Beam theory}

Equations (1) and (2) given by Peirce are the solution to a differential equation expressing the equilibrium of a small part of a beam under large bending deformations. ${ }^{20,22}$ This differential equation is written as

$$
\frac{d^{2} \phi}{d s^{2}}=-s \cos \phi / c^{3}
$$

In Equation (4), $s$ represents the distance of a point $P$ along the strip from the free end, $\phi$ the angle between the tangent there and the horizontal and $c=(G / w)^{1 / 3}$, as shown in Figure 2.

Peirce $^{20}$ states that there is no analytical solution available to this differential equation. Instead, an expansion of $\phi$ in a power series was used to approximate the solution. This laborious work was performed by Hummel and Morton, ${ }^{22}$ who actually focused on solving the bending of thin flexible strips and used a pen-steel strip as an example, illustrating that the method can be used for any type of material and is not limited to fabric materials. Based on the calculations of Hummel and Morton, a smooth curve is fitted to the data:

$$
G=w \cdot l \cdot \frac{\cos \theta / 2}{8 \cdot \tan \theta}
$$

Equation (5) is the known formula stated above, and - after application of a correction factor - used by ASTM as a standard for testing. However, Peirce ${ }^{20}$ contains a warning stating "for satisfactory accuracy more terms are necessary in the expansion, but..." before going on to Equation (5). This warning is even more clear in Hummel and Morton, ${ }^{22}$ where the reader is warned that the usability of the expansion is only accurate for small angles, and the power series expansion requires many more terms for larger angles (such as $\theta=41.5^{\circ}$ in ASTM).

Nowadays, computing power allows the accurate solving of differential equations through numerical techniques. The solving technique described by Hummel and Morton ${ }^{22}$ was implemented in MATLAB, using an ordinary differential equation (ODE) solver to find the solution rather than relying on the previously discussed power series. Using a standard computer, solving the equation for a range of $\theta=1 \ldots 70^{\circ}$ in steps of $0.5^{\circ}$ takes less than $60 \mathrm{~s}$, and therefore does not hinder the practical usability of this technique.

\section{Compensation for plate effects}

The finite element results shown in Figure 4 illustrate that plate effects have an influence on the bending behavior of the sample under investigation. Due to Poisson effects, the cross-section of the foil will warp in regions of high curvature, resulting in a locally 


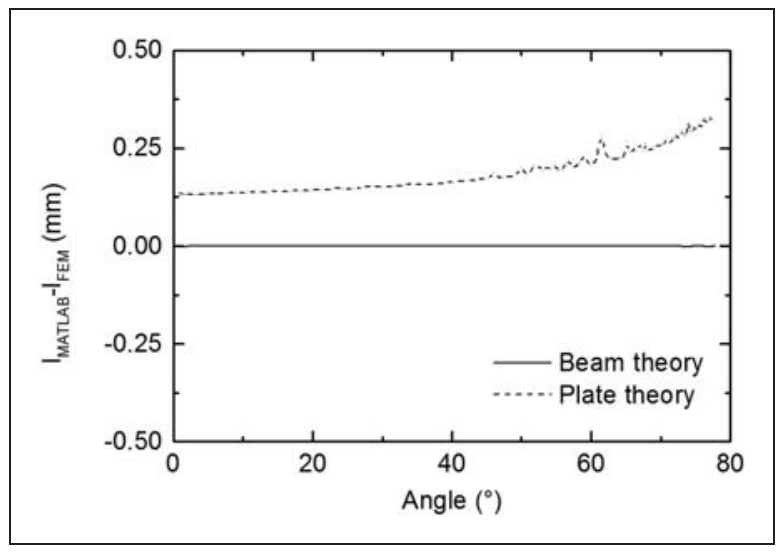

Figure 5. Difference in predicted overhanging length between ordinary differential equation technique and finite element for plate and beam theory.

increased inertial moment, thereby resulting in a stiffer response as illustrated in Figure 4.

Compensating exactly for these plate effects would require solving of large-deflection cantilever plate equations. This would increase the computational difficulties tremendously and would severely hinder the practical use of the method presented.

Therefore, it is chosen to use the flexural rigidity definition from small deflection linear-elastic plate theory, while using the differential equation in Equation (4):

$$
G=\frac{E \cdot t^{3}}{12 \cdot\left(1-v^{2}\right)}
$$

Figure 5 shows the difference in calculated overhanging length between finite element simulations and the ODE-technique described for both beam theory $(G=E \cdot I)$ and plate theory (Equation (6)).

As can be seen from Figure 5 there is a near-perfect correspondence between the ODE technique and finite element implementation when beam theory is utilized. A somewhat larger difference exists when plate theory is used, which is to be expected considering that the plate equations were not solved numerically in this approach, and a simplified methodology was used. Nonetheless, at $\theta=41.5^{\circ}$, the error between the ODE technique and finite element is $0.16 \mathrm{~mm}$ using plate theory (and just $30 \mathrm{~nm}$ using beam theory). It is more than likely that the measurement accuracy and repeatability of the cantilever test will exceed this value and therefore these errors can be neglected.

\section{Implications of different solving techniques}

Up to now, the results have only focused on the $l-\theta$ relationship, showing that different techniques lead to
Table 2. Calculated E-modulus based on different techniques.

\begin{tabular}{lrrrr} 
& ASTM & Peirce & \multicolumn{2}{l}{ ODE technique } \\
\cline { 3 - 5 } & & & $\begin{array}{l}\text { Beam } \\
\text { theory }\end{array}$ & $\begin{array}{l}\text { Plate } \\
\text { theory }\end{array}$ \\
\hline E-modulus (GPa) & 13.076 & 9.027 & 8.536 & 8.002 \\
Deviation (\%) & $+62.6 \%$ & $+12.25 \%$ & $+6.14 \%$ & $-0.5 \%$ \\
\hline
\end{tabular}

ODE: ordinary differential equation; ASTM: American Society for Testing and Materials.

a different relationship. Assuming that the finite element model using plate theory is the most accurate technique, we find that for a Upilex-50S foil as defined previously an overhang length of $99.5668 \mathrm{~mm}$ is necessary to achieve the angle $\theta=41.5^{\circ}$. Using this length as a starting point, we can now compare the predicted E-modulus using the different techniques discussed. The results of this analysis are gathered in Table 2 .

The results in Table 2 clearly illustrate the increased accuracy of the proposed ODE techniques over the currently available methods. Based on the data in Table 2, the ASTM standard severely overestimates the E-modulus (and correspondingly, the bending stiffness). While Peirce provides a much better prediction, a lot of accuracy can still be gained by implementing the ODE methodology. Even assuming beam theory (when the Poisson's ratio is unknown), significant improvements can be achieved.

\section{Experimental results}

In order to validate the improved accuracy of the presented method over the current standard method, a series of experimental tests have been conducted. While comparisons between different techniques can be made for any type of material (e.g. fabrics), statements about absolute accuracy can only be made when a reference value is known. Using fabric materials, the bending stiffness stated by the manufacturer would most likely be based on the ASTM standard discussed in this work. This would inevitably result in the incorrect conclusion that the ASTM standard achieves the most accurate results. As a consequence only isotropic materials can be used. The bending stiffness of continuous, isotropic materials can be related to the in-plane tensile modulus, which can be measured by independent tensile tests. For the purpose of this work, tests were performed on Upilex-50S foil.

\section{Sample specifications}

In accordance with the ASTM standard, five samples of Upilex-50S foil were cut along the material direction 


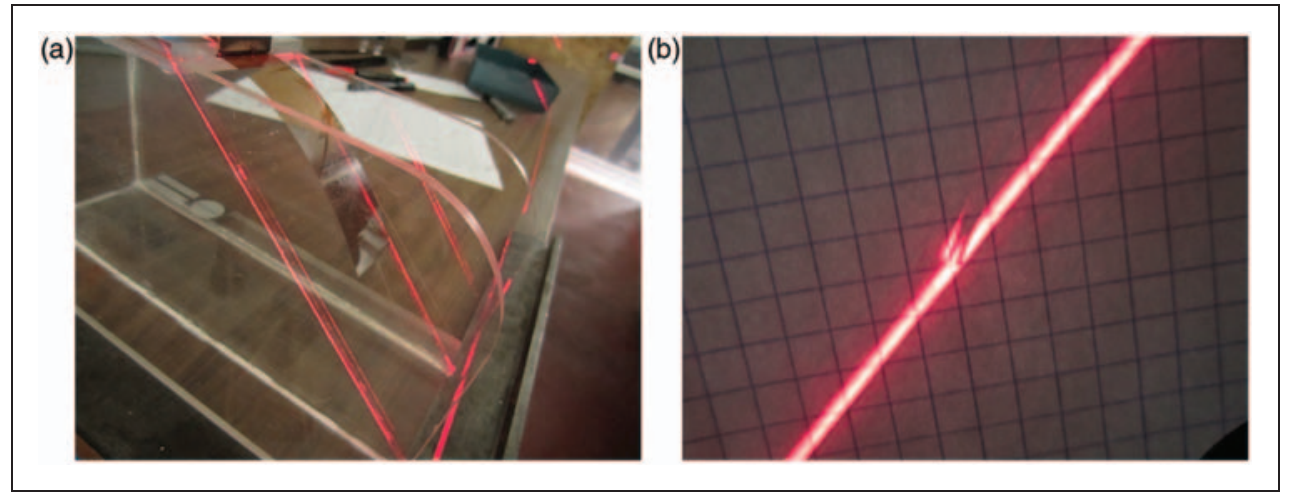

Figure 6. (a) Peirce cantilever test device with aligned laser plane. (b) Projection onto a sheet of paper showing Upilex-50s foil crossing the laser plane.

(MD) and five perpendicular to the MD. The width of each sample was measured to an accuracy of $0.05 \mathrm{~mm}$. The edges were found to be parallel to within $0.5 \%$. The thickness was measured at several locations on each sample to an accuracy of $0.001 \mathrm{~mm}$. Thickness variations were found to be less than $3 \%$.

\section{Test set-up}

Cantilever test. The samples were first tested in a commercial cantilever test device as required by the ASTM D1388 standard. The device was made out of transparent Plexiglas with an etched line inclined at $41.5^{\circ}$ on each face of the set-up. In order to improve the accuracy of the measurements, a laser plane was aligned precisely with the inclined lines on the test device (Figure 6(a)). This allowed for a much more precise determination of overhanging length to achieve precisely the desired angle of $41.5^{\circ}$ (Figure 6(b)). For each sample, the face and back of both ends were tested, leading to a total of four measurements per sample.

Tensile test. After the cantilever test, the tensile modulus was determined according to ASTM D882. The tests were performed immediately after the cantilever test at the same environmental conditions. In order to prevent slippage in the grips, rubber padding was used. The tests were performed at the required rate of 25 $\mathrm{mm} / \mathrm{min}$ and the entire load-displacement curve was recorded for each sample. All samples were loaded up to fracture and showed a clean break in the mid-section of the sample.

\section{Results}

The results of the tensile test are shown in Table 3. A distinction is made between samples parallel to the MD and those perpendicular to it because a difference in
Table 3. Tensile modulus for Upilex-50S foil according to ASTM D882.

\begin{tabular}{ll}
\hline & E-modulus \\
\hline$\| M D(M P a)$ & $9191 \pm 341$ \\
$\perp M D(M P a)$ & $8214 \pm 303$ \\
\hline
\end{tabular}

stiffness for both directions was found. The tensile modulus was determined as described in the ASTM D882 standard.

As was done with the tensile tests, the results for the cantilever test are separated into samples parallel to the MD and those perpendicular to it. The four measurements per sample were averaged before calculating the bending stiffness of a single sample. For each sample, the tensile modulus was determined according to ASTM, Peirce and both ODE approaches. The averaged values over all samples in a certain direction are given in Table 4 and compared to the reference values given in Table 3.

\section{Discussion of results}

The results in Table 4 clearly show the improved accuracy of the proposed method over those currently available. Even under the assumptions of beam theory, the MATLAB implementation shows clear improvements over both Peirce and ASTM formulations. As was already shown previously, the ASTM standard provides a poor estimate of actual E-modulus and bending stiffness. The original formulation by Peirce achieves better results, although errors up to $18 \%$ can be found for the parallel samples.

In the parallel samples, the ODE plate theory implementation slightly underestimates the E-modulus as found through tensile testing. This is to be expected 
Table 4. Tensile modulus for Upilex-50S foil based on the cantilever test.

\begin{tabular}{lccll}
\hline$\|$ MD & ASTM & Peirce & \multicolumn{2}{l}{ ODE technique } \\
\cline { 4 - 5 } & & & Beam theory & Plate theory \\
\hline E-modulus $(\mathrm{MPa})$ & 14,837 & 10,243 & 9686 & 9080 \\
& $( \pm 724)$ & $( \pm 500)$ & $( \pm 473)$ & $( \pm 443)$ \\
Error & $+61 \%$ & $+11 \%$ & $+5 \%$ & $-1 \%$ \\
\hline$\perp$ MD & ASTM & Peirce & ODE technique & \\
\cline { 5 - 5 } & & & Beam theory & Plate theory \\
\hline E-modulus $(\mathrm{MPa})$ & 14,093 & 9729 & 9200 & 8625 \\
Error & $( \pm 734)$ & $( \pm 507)$ & $( \pm 479)$ & $\begin{array}{l}( \pm 449) \\
+72 \%\end{array}$ \\
\hline
\end{tabular}

ODE: ordinary differential equation; ASTM: American Society for Testing and Materials.

due to the simplified technique used to compensate for plate effects. The perpendicular samples, on the other hand, result in an overestimation of the E-modulus. Because the Upilex-foil was stored on a roll, the foil material had a small (yet noticeable) pre-curvature. This small curvature is averaged out in the parallel samples since measurements on both face and back ends were made. In the case of the perpendicular samples, however, the pre-curvature leads to a slightly curved cross-section with an increased moment of inertia resulting in a stiffer response of the foil in cantilever tests. Since this curvature is not accounted for in the ODE implementation, this inevitably leads to an overestimation of the E-modulus. Nonetheless, even without compensating for this effect the correspondence between the ODE implementation and the stiffness determined through tensile testing is very good.

\section{Conclusions}

This work has illustrated that the methodologies described by Peirce and the American Society for Testing and Materials (ASTM) do not correspond to the results of finite element simulations. The origins of this discrepancy were traced back and a modified technique was proposed using numerical solving of the governing differential equations. It was shown theoretically that these modifications lead to results that correspond perfectly to those predicted by finite element beam theory. In addition, finite element simulations revealed the importance of plate effects in the samples, which should be accounted for if accurate measurements are to be achieved. The proposed methodology was adapted to account for these effects, showing only minimal differences between finite element results and the new methodology.
The usability of the method was shown to not only produce theoretical increases in accuracy, but also measurable improvements by a series of experimental tests on Upilex-50S foil. The results show that the new methodology is capable of measuring the E-modulus to within $1 \%$ of the reference value, while Peirce $(+11 \%)$ and $\operatorname{ASTM}(+61 \%)$ produce far less accurate results.

The proposed method produces results that are comparable to finite element predictions. However, in order to use finite element software to determine bending stiffness based on a given overhang length, an iterative process would be needed increasing computational efforts and time, hindering the practical usability of finite element simulations. Using standard computer equipment, the proposed methodology requires less than $60 \mathrm{~s}$ to determine the flexural rigidity based on the overhanging length and therefore does not limit the practical usability.

The presented model is found to produce more accurate results than currently available tests at minimal computational effort, and consequently enables the more accurate simulation of composite forming processes.

\section{Funding}

The research leading to these results has received funding from the European Union Seventh Framework Programme FP7/2007-2013 (grant agreement $n^{\circ} 257733$ (SmartFiber)).

\section{References}

1. Long MJ. Composite forming mechanisms and materials characterisation. In: Long AC (ed.) Composites forming technologies. Cambridge, England: Woodhead Publishing in Textiles, 2007.

2. Lim TC and Ramakrishna S. Modelling of composite sheet forming: a review. Compos Appl Sci Manuf 2002; 33: 515-537. 
3. Cherouat A and Bourouchaki H. Numerical tools for composite woven fabric preforming. Adv Mater Sci Eng 2013; 1-18.

4. Vanclooster K, Lomov SV and Verpoest I. Experimental validation of forming simulations of fabric reinforced polymers using an unsymmetrical mould configuration. Compos Appl Sci Manuf 2009; 40: 530-539.

5. Cherouat A and Borouchaki H. Present state of the art of composite fabric forming: geometrical and mechanical approaches. Materials 2009; 2: 1835-1857.

6. Golden K, Rogers TG and Spencer AJM. Forming kinematics of continuous fibre reinforced laminates. Compos Manuf 1991; 2: 267-277.

7. Mack C and Taylor HM. 39 - the fitting of woven cloth to surfaces. J Textil Inst Trans 1956; 47: 477-488.

8. Trochu F, Hammami A and Benoit Y. Prediction of fibre orientation and net shape definition of complex composite parts. Compos Appl Sci Manuf 1996; 27: 319-328.

9. Bergsma O. Three-dimensional simulation of fabric draping. Delft: Delft University of Technology, 1995.

10. Badel P, Gauthier S, Vidal-Salle E, et al. Rate constitutive equations for computational analyses of textile composite reinforcement mechanical behaviour during forming. Compos Appl Sci Manuf 2009; 40: 997-1007.

11. Dong L, Lekakou $C$ and Bader MG. Solid-mechanics finite element simulations of the draping of fabrics: a sensitivity analysis. Compos Appl Sci Manuf 2000; 31: 639-652.

12. Hamila $\mathrm{N}$ and Boisse P. A meso-macro three node finite element for draping of textile composite preforms. Appl Compos Mater 2007; 14: 235-250.

13. Hamila N, Boisse P, Sabourin F, et al. A semi-discrete shell finite element for textile composite reinforcement forming simulation. Int J Numer Meth Eng 2009; 79: $1443-1466$.
14. Chen BJ and Govindaraj M. A physically-based model of fabric drape using flexible shell theory. Textil Res J 1995; 65: 324-330.

15. Kang TJ and Yu WR. Drape simulation of woven fabric by using the finite-element method. $J$ Textil Inst 1995; 86: 635-648.

16. Yu WR, Harrison $\mathrm{P}$ and Long A. Finite element forming simulation for non-crimp fabrics using a non-orthogonal constitutive equation. Compos Appl Sci Manuf 2005; 36 : 1079-1093.

17. Boisse P. Finite element analysis of composite forming. In: Long AC (ed.) Composites forming technologies. Cambridge, England: Woodhead Publishing in Textiles, 2007, pp.46-75.

18. Jauffrès D, Morris C, Sherwood J, et al. Simulation of the thermostamping of woven composites: mesoscopic modelling using explicit fea codes. Int J Mater Forming 2009; 2: $173-176$.

19. Young M and Paton R. Diaphragm forming of resin preimpregnated woven carbon fibre materials. In: Falcone A, Nelson KM, Albers R, et al (eds) Advancing affordable materials technology. Covina, CA: Society for the Advancement Material \& Process Engineering, 2001, pp.551-563.

20. Peirce FT. 26-the "handle" of cloth as a measurable quantity. J Textil Inst Trans 1930; 21: T377-T416.

21. ASTM D1388-08:2008. Standard test method for stiffness of fabrics.

22. Hummel FH and Morton WB. XXXII. On the large bending of thin flexible strips and the measurement of their elasticity. Philos Mag Series 7. 1927; 4: 348-357.

23. Szablewski P and Kobza W. Numerical analysis of Peirce's cantilever test for the bending rigidity of textiles. Fibres Textil East Eur 2003; 11: 54-57. 